\section{Biofunctionalized Magnetic Microdiscs Achieve Cancer Cell Destruction}

Recently, magnetic nanoparticles have been considered as a novel and promising means of fighting cancer. Magnetic nanoparticles serve as drug carriers which are released into the blood stream and come under the influence of a targeting magnetic field, which direct them onto tumor-sites until the drug gets completely released. These treatments have been successful in animals and are being considered for clinical trials. However, agglomeration of nanoparticles has raised serious concerns as they might occlude blood vessels, limiting their suitability for therapy. As described in the February issue of Nature Materials (DOI: 10.1038/ NMAT2591; p. 165), D.-H. Kim of the Materials Science Division of Argonne National Laboratory, E.A. Rozhkova of the Center for Nanomaterials at Argonne, I.V. Ulasov of the Brain Tumor Center of the University of Chicago Pritzker School of Medicine, and their colleagues have successfully interfaced lithographically defined ferromagnetic materials that have a spin vortex ground state with cancer cells. The researchers demonstrated that the magnetic

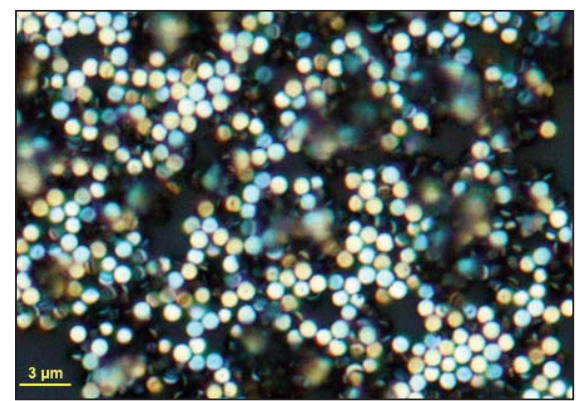

Figure 1. Optical micrograph of lithographically defined $\sim 1 \mu \mathrm{m}$ diameter 20:80\% iron-nickel microdiscs (MDs), coated with a 5-nm-thick layer of gold.

spin vortex-induced magneto-mechanical stimulus leads to cancer cell membrane damage, as well as to cellular signal transduction and amplification, causing initiation of apoptosis, also known as programmed cell death or cell suicide. Manifestation of apoptosis is of clinical significance because the malignant cells are known to be almost "immortal" due to suppressed apoptosis and, consequently, highly resistant to conventional chemo/ radiotherapies.
The researchers achieved a more precise control of disc geometry and their magnetic properties using lithography which is challenging with conventional wet chemistry routes. Specifically, they prepared $60 \mathrm{~nm}$ thick, $\sim 1 \mu \mathrm{m}$ diameter 20:80\% iron-nickel microdiscs (MDs), coated with a 5-nm thick layer of gold by optical lithography and magnetron sputtering (see Figure 1). Specific targeting of MDs to cancer cells was achieved by functionalization with an antibody (mAb) to the IL13 $\alpha 2$ receptor which served as a marker over-expressed by cancer, but not healthy cells. The research team found that biorecognition properties of the free $\mathrm{mAb}$ were preserved in the MD-anti-IL13 $\alpha 2 \mathrm{R}$ hybrid material. The researchers investigated the dynamics of MDs in an aqueous environment by applying an alternating magnetic field (ac) which caused shifts in the microdisc vortices, creating an oscillation that transmitted a mechanical force to the cancer cell. Loss of cell-membrane integrity was identified using various assays and optical microscopies, which confirmed the magnetic field induced cell death using MD-mAb.

Interestingly, with the use of low frequencies $(10 \mathrm{~Hz}$ and $20 \mathrm{~Hz})$, the re-

\section{Hall Effect Measurement Systems}

$\diamond \quad$ Turnkey Systems

$\diamond \quad$ Configurable and Modular Systems

$\diamond \quad$ Automated Instrumentation

$\diamond \quad$ Wide Temperature Ranges Available

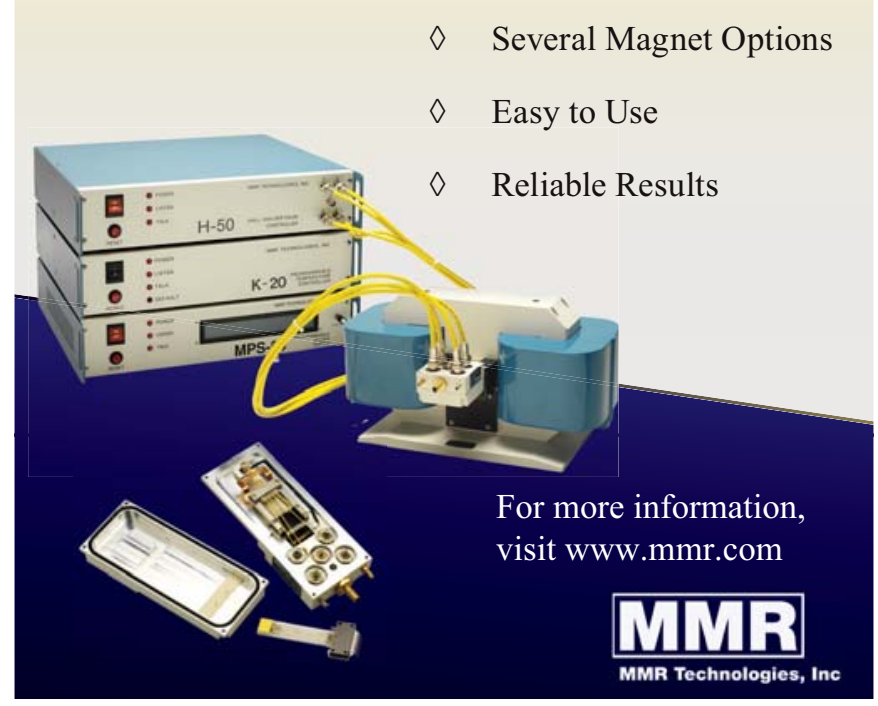

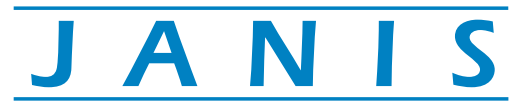

\section{Cryogenic Systems}

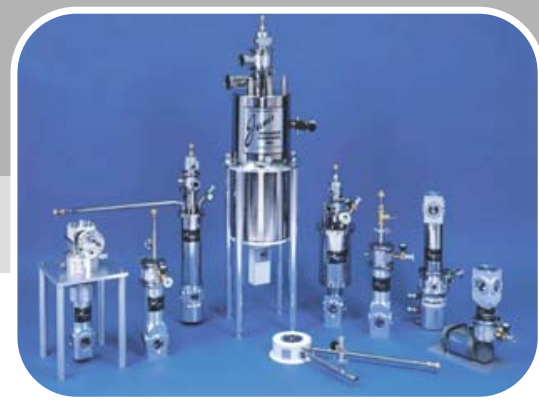

Does your research require low temperatures? Contact Janis today. Our engineers will assist you in choosing the best system for your application.
$10 \mathrm{mK}$ to $800 \mathrm{~K}$
Cryocoolers
Magnet Systems
LHe/LN 2 Cryostats
- Dilution Refrigerator Systems
Micro-manipulated Probe Stations 
searchers achieved $90 \%$ cancer cell destruction. Their optical micrographs clearly indicated the rounded cells with membrane shrinkage and loss of membrane integrity under an ac magnetic field of $90 \mathrm{Oe}$ at a frequency of $20 \mathrm{~Hz}$. Through atomic force microscopy measurements and nuclear DNA scission assays results, the researchers hypothesized that membrane rupture alone does not appear to play a dominant role in cancer cell destruction; instead, applied magnetic field to functionalized MD-cell complexes can possibly lead to triggering of intracellular pathways leading to programmed cell death.

The researchers emphasized that these magnetic nanoparticles demonstrate a unique combination of multifunctional properties as they have zero moment in remanence, possess high value of the magnetization of saturation, and can be easily biofunctionalized. The researchers also said, "magnetic microdiscs with a spin-vortex ground state may find further application in distant cell manipulation and separation, controllable force application during regenerative tissue growth, and studies of elasticity in a diagnostic capacity."

Co-authors of the article are S.D. Bader, T. Rajh, M.S. Lesniak, and V. Novosad.

ROHIT KHANNA

\section{Two-Step Microfluidics Approach Enables Electronic Detection of Biomarkers in Blood}

E. Stern, M.A. Reed, and T.M. Fahmy of Yale University, and their colleagues have produced microfluidic device components capable of detecting picomolar levels of cancer biomarkers in whole blood. In the December 13, 2009 online issue of Nature Nanotechnology (DOI: 10.1038/NNANO. 2009.353), the research team describes a two-step process designed to circumvent the downside of a nanowire sensors' extreme sensitivity. Previously, researchers have shown that nanowire field-effect transistors (FETs) functionalized with anti- bodies can detect the presence of antigen at clinically relevant picomolar concentrations, but in complex physiological solutions such as blood the ultrasensitive devices are quickly fouled and lose their utility. This has previously limited clinical applications for the technology.

Now, Stern and his colleagues overcome this problem by introducing an upstream microfluidic purification chip (MPC) that captures the antigen of interest, isolates it from other blood components, and releases the antigen into a buffer suitable for use with nanowire sensing. The MPC contains numerous microscopic pillar features, produced by lithographic techniques, that are functionalized with antibodies against prostate-specific antigen (PSA) or carbohydrate antigen 15.3 (CA15.3), commonly used biomarkers for prostate and breast cancer, respectively. The antibodies are attached to the pillars through photo-labile linkers. The antibodies capture their antigens out of whole blood and retain them within the MPC while the other blood components are washed out. After washing, a sensor-compatible buffer is introduced into the MPC and UV light is used to cleave the antigens and attached antibodies from the pillars. The buffer and its load of antigen-antibody complexes can then be transferred to a separate microfab-

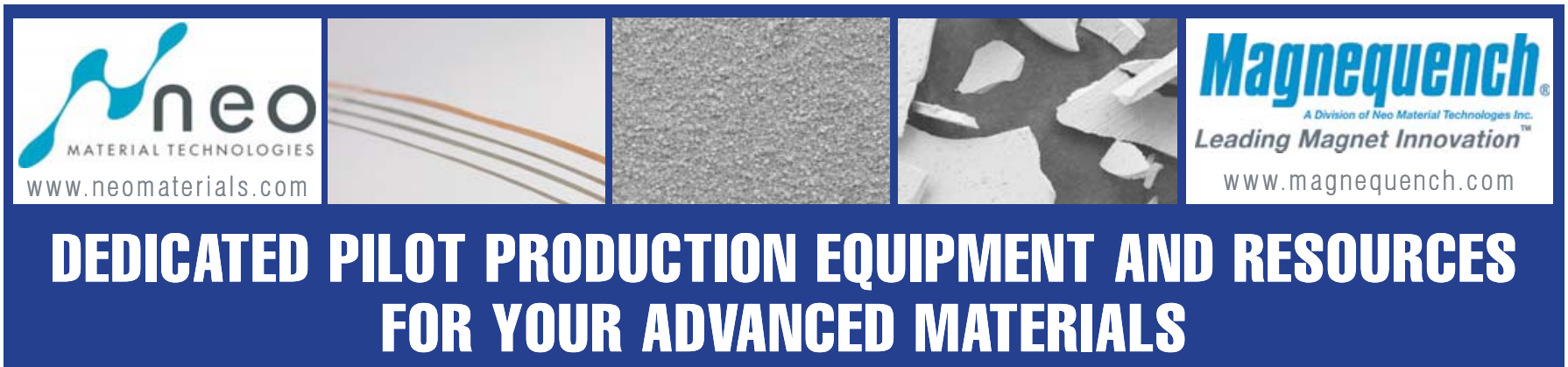

Powder Motallurgy • Rapid Solidification · Specialty Alloys Melt Spinning
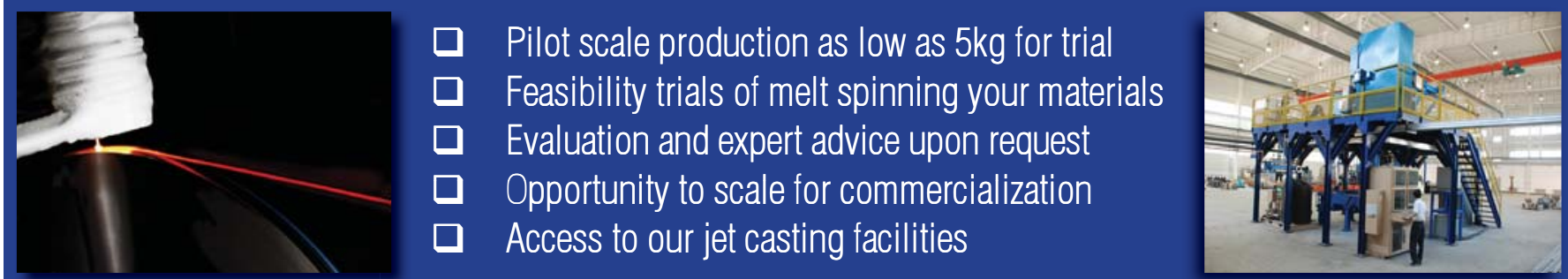

We Specialise in

Post-casting heat treatment Handling and processing powder Processing ribbon, foil and powder Melt spinning in vacuum and inert atmosphere Rapid quenching to generate nanocrystalline and amorphous alloys

We are the World's Largest Melt Spinner of Specialty Alloys
61 Science Park Road, \#01-19 The Galen, Singapore Science Park II, Singapore 117525.
research@magnequench.com
Iso 14001:2004 and ISO 9001:2000 Certified

